\title{
The Contrasting Role of Water and Acid Within Organic Phase Amphiphile Aggregation
}

\author{
Biswajit Sadhu ${ }^{*, \dagger, \ddagger}$ and Aurora E. Clark ${ }^{*, \dagger, \uparrow, \S}$ \\ †Department of Chemistry, Washington State University, Pullman, WA 99164 \\ $\ddagger$ Health Physics Division, Bhabha Atomic Research Centre, Mumbai, India \\ \ Voiland School of Chemical Engineering and Bioengineering, Washington State \\ University, Pullman, WA 99164 \\ $\S$ Pacific Northwest National Laboratory, Richland, WA 99354 \\ E-mail: bsadhu@barc.gov.in, biswajit.sadhu@wsu.edu; auclark@wsu.edu
}




\begin{abstract}
The self-assembly of amphiphiles is often modified by the presence of co-solutes and significant study has examined this behavior in aqueous systems. Much less is known about the role of polar co-solutes upon amphiphile aggregation within nonpolar media, however such conditions are relevant to a variety of industrial processes not the least of which are separations systems like those found in liquid-liquid extraction (LLE). Therein, surface active amphiphiles extract water, acid, and other solutes of interest. Intriguing increases to amphiphile aggregates have been experimentally observed upon water and acid extraction, however a myriad of competitive intermolecular interactions have thus far prevented a fundamental understanding of the individual and dual role of these solutes upon amphiphile self-assembly. Toward this end, this work employs classical molecular dynamics and graph theory analyses to deconstruct the individual affects of water and nitric acid upon the self-assembly of $\mathrm{N}, \mathrm{N}, \mathrm{N}^{\prime}, \mathrm{N}^{\prime}-$ tetraoctyl-3-oxapentanediamide (TODGA), a prevalent amphiphile extractant used in metal ion separations. In the absence of acid, and at low water concentration, $\mathrm{H}_{2} \mathrm{O}$ is found to promote local dimer and trimer formation of TODGA, however as $\left[\mathrm{H}_{2} \mathrm{O}\right]_{\text {org }}$ increases, the preferential solvation of water with itself causes the formation of large water clusters that serve to link large TODGA clusters on the periphery (causing extended aggregation). Addition of $\mathrm{HNO}_{3}$ to the humid solutions disrupts the water hydrogen bond network and inhibits the formation of large water clusters - thus preventing extended aggregation behavior. We rationalize the prior experimental observations as being attributed primarily to the role of water in the self-assembly of TODGA rather than co-extracted $\mathrm{HNO}_{3}$, thus providing valuable new insight into the means by which extractant aggregation can be tuned within LLE processes. In addition, this work differentiates the role of polar solutes upon amphiphile self-assembly via their individual hydrogen bonding capabilities and competitive interactions that disrupt preferred solvation environments.
\end{abstract}




\section{Introduction}

Self-assembly of amphiphilic molecules is a key characteristic of many systems with wide ranging applicability, not limited to polymers, ${ }^{1-3}$ proteins, ${ }^{4}$ and catalysts. ${ }^{5}$ In synthetic systems involving polymers or surfactants, this ubiquitous process is the basis for the formation of polymeric vesicles and micelles that are essential to drug delivery devices, ${ }^{6,7}$ and also in biphasic purification strategies. ${ }^{8}$ Within the separations of complex aqueous mixtures via liquid-liquid extraction (LLE), amphiphilic extractants are often employed to selectively partition specific solutes from the aqueous into the organic phase. This is generally the approach taken with metal mixtures, and in this case such amphiphiles also partition other polar solutes, including water and acids. The self-assembly of these amphiphiles leads to a wide range of structures that often determines the efficiency of the separations process (influencing the distribution coefficients and selectivity of solutes of interest) as their interactions with solutes influence the extraction free energy. ${ }^{9}$ The concentration of amphiphile aggregates is incredibly important, as at high concentrations they contribute to undesirable phase splitting, ${ }^{10}$ but at moderate concentrations they can increase extraction efficiency presumably because the aggregates themselves are better extractants than the individual extractant-solute (or metal-ligand, ML) complexes. ${ }^{11}$ Recent studies have further implied that the organizational structure of aggregates, their diversity in volume, stoichiometry, and associated micro-structural features may have strong influence on the extraction event and trigger a synergistic effect if multiple extractants are employed. ${ }^{12,13}$

Decoupling the separate influence of water and acid upon aggregation is a significant technical challenge within experimental LLE because both water and acid are often ubiquitious in the organic phase (even if at low concentration). ${ }^{14,15}$ The water concentration in the organic phase is not only dependent on the characteristics of the amphiphile extractant but also on the specific acid. ${ }^{16}$ While $\mathrm{HNO}_{3}$ is known to increase water concentration in organic media, other strong acids like $\mathrm{HCl}$ do not have this effect. ${ }^{16}$ At the same time, the acid extraction in the organic phase depends upon its individual interactions with both 
metal-extractant complexes and free extractant amphiphile molecules. ${ }^{17}$ These dependencies often ensure the simultaneous presence of water and acid in the organic medium albeit at varying concentration. As such, aggregation mechanisms are the result of complex coupled interactions that can be considered as an amphiphile-water-acid network (as supported by $\left.\operatorname{FTIR}^{18}\right)$.

LLE systems are a useful platform to consider the role of varying hydrogen bond capabilities of polar solutes within the self-assembly of amphiphiles in non-polar media - both in the context of size and composition of the resulting aggregates, but also upon the intermolecular interaction patterns that govern their morphology. There remains significant need for molecular-scale analysis of the sensitivity of amphiphile assembly processes to the solution composition, coupled to rigorous study of the correlating relationships of aggregate composition, size, morphology. Molecular dynamics is a complementary method for understanding the self-assembly of amphiphiles,,${ }^{19,20}$ where recent developments of graph theoretical (network) methods have paved the way for discovering key relationships between network structure and the physicochemical properties of many systems. ${ }^{21,22}$ In the present work, we have investigated the critical role of water and acid within the aggregation of an amphiphilic extractant using molecular dynamics in combination with network analyses. A solvent extraction system consisting of $\mathrm{N}, \mathrm{N}, \mathrm{N}^{\prime}, \mathrm{N}^{\prime}$-tetraoctyl-3-oxapentanediamide (TODGA) in $n$-dodecane has been examined due to its relevance within actinide and lanthanide separations within the nuclear fuel cycle (notably the Actinide Lanthanide $\underline{\text { SEParation Process }}$ $($ ALSEP) $) .{ }^{23}$ Further, this amphiphile can exhibit phase splitting behavior at moderate acid concentration. ${ }^{24}$ The acid driven aggregation of TODGA, even in the absence of metal, is a well-known phenomena and has been experimentally studied using small-angle neutron scattering (SANS) and vapor-pressure osmometry (VPO). ${ }^{11,25,26}$

The available experimental data and diverse aggregation behavior of TODGA is ideal for developing a platform of basic insight into the varying hydrogen bond (HB) capabilities of polar solutes upon amphiphile aggregation in non-polar media. Here, we demonstrate that 
$\mathrm{H}_{2} \mathrm{O}$ and $\mathrm{HNO}_{3}$ have different mechanistic roles that facilitate amphiphile self-assembly where clear differentiation is observed upon the resulting aggregate size distribution, composition, and morphology. As water concentration is increased within an organic solution the dual hydrogen bond accepting and donating capabilities cause a transition from $\mathrm{H}_{2} \mathrm{O}$ acting as an individual bridging constituent between TODGA molecules, to preferential solvation that causes larger water clusters to be formed whose surfaces bring together TODGA clusters that form extended aggregate assemblies. Addition of $\mathrm{HNO}_{3}$ to the humid organic solutions disturbs the preferential self-solvation of water by rearranging its HB network to participate in the multiple $\mathrm{HB}$ acceptor sites of $\mathrm{HNO}_{3}$. This in turn inhibits the formation of large water clusters that support TODGA aggregate-aggregate merging. We anticipate that these mechanistic insights will help achieve better control over amphiphile self-assembly across length-scales and may assist process layouts that leverage such aggregation as in solvent extraction, polymer synthesis, drug-delivery and catalysis.

\section{Computational and Analysis Methods}

\section{Simulation Protocol}

System Composition. Table 1 presents the composition of all simulated systems, with different conditions labelled A - C. System A corresponds to 0.1 M TODGA dissolved in $n$-dodecane, and is based the experimental critical micelle concentration (CMC) of TODGA at $2 \mathrm{M} \mathrm{HNO}_{3(a q)}$ and $25^{\circ} \mathrm{C}{ }^{25,26}$ The B-series of systems introduce water to TODGA/ndodecane, where the water concentration is gradually increased from B1 (0.02 M) to B2 $(0.05 \mathrm{M})$ to $\mathbf{B 3}\left(0.2 \mathrm{M} \mathrm{H}_{2} \mathrm{O}\right)$. In the $\mathbf{C}$-series, nitric acid is introduced to water-containing

conditions. The $\mathbf{C 1}$ and $\mathbf{C 2}$ systems correspond to prior experimental studies that have 0.02 $\mathrm{M}$ and $0.05 \mathrm{M} \mathrm{HNO}_{3}{ }^{11,25,26}$ and the same water content as $\mathbf{B} 1$ and $\mathbf{B 2}$, respectively. The C3 - C5 series have the $0.20 \mathrm{M} \mathrm{H}_{2} \mathrm{O}$ content of B3 and varying $\left[\mathrm{HNO}_{3}\right]$ up to $0.15 \mathrm{M}$. 
Table 1: Compositions of simulated systems

\begin{tabular}{cccc}
\hline Conditions & TODGA $(\mathrm{M})^{\mathrm{a}}$ & Water $_{(\mathrm{M})^{\mathrm{a}}}$ & Nitric Acid $(\mathrm{M})^{\mathrm{a}}$ \\
\hline $\mathbf{A}$ & $0.10[60]$ & - & - \\
\hline B1 & $0.10[60]$ & $0.02[12]$ & - \\
B2 & $0.10[60]$ & $0.05[30]$ & - \\
B3 & $0.10[60]$ & $0.20[120]$ & - \\
\hline C1 $^{\mathbf{b}}$ & $0.10[60]$ & $0.02[12]$ & $0.01[6]$ \\
C2 $^{\mathbf{c}}$ & $0.10[60]$ & $0.05[30]$ & $0.05[30]$ \\
C3 & $0.10[60]$ & $0.20[120]$ & $0.01[6]$ \\
C4 & $0.10[60]$ & $0.20[120]$ & $0.05[30]$ \\
C5 & $0.10[60]$ & $0.20[120]$ & $0.15[90]$
\end{tabular}

${ }^{a}$ Values in bracket correspond to the number of molecules present in the simulation. ${ }^{b}$ Experimental conditions of Nave et al..$^{25}$ Experimental conditions of Yaita et al. ${ }^{11}$

Force Field Implementation. The interactions of $n$-dodecane was taken from Vo et al. ${ }^{27}$, while the force field of TODGA was generated by the Generalized AMBER Force Field $(\text { GAFF2 })^{28}$ parametrization using the geometry-optimized structure of TODGA using density functional theory (DFT) with the B3LYP ${ }^{29,30}$ functional and 6-31g* basis set. ${ }^{31}$ The restrained electrostatic potential (RESP) approach was employed to derive the partial charges. This protocol follows a similar approach to that of Hirata et al. ${ }^{32}$, who employed the AMBER force field parameters with RESP-fitted charges to investigate the complexation of $\mathrm{Ln}^{3+}$ and $\mathrm{UO}_{2}{ }^{2+}$ with a tetra-methyl DGA extractant (TMDGA). As part of the benchmarking process we note that the employed parameters reproduce the correct density of 0.1 M TODGA in dodecane as (predicted to be $0.788 g L^{-1}$ relative to the experimental value of $0.759 g L^{-133}$ ), and measured diffusion coefficient ${ }^{34}$ (see Results section and Supporting Information). The TIP3P water model ${ }^{35}$ was used along with the $\mathrm{HNO}_{3}$ parameters of Servis et al. ${ }^{21}$ which uses the molecular (undissociated) form that is congruent with experimental $\operatorname{IR}^{36}$ and $a b$ initio theoretical predictions. ${ }^{16,37}$ Additional details regarding the force field parameters are provided in the Supporting Information (cf. Figure S1, Table S1-S4).

Molecular Dynamics Simulations. The initial system configurations were generated using Packmol ${ }^{38}$ by distributing all molecules randomly in a cubic box of size $10 \times 10 \times 10$ 
$\mathrm{nm}^{3}$. The volume of diluent was corrected considering the equivalent volume of replacement of added TODGA, $\mathrm{H}_{2} \mathrm{O}$ and $\mathrm{HNO}_{3}$. The simulation box constitutes a representation of the organic phase and no interface was generated during equilibration. Subsequent molecular dynamics simulations were performed using GROMACS 2019.4. ${ }^{39}$ Each system was first energy-minimized using steepest descent, then subjected to 20 ns of NPT molecular dynamics at $300 \mathrm{~K}$ and 1 bar using the Berendsen barostat ${ }^{40}$ with a 2 fs time step. This was followed by a further 20 ns equilibration in NVT with Nose-Hoover thermostat ${ }^{41}\left(\tau=0.4 \mathrm{ps}^{-1}\right)$, followed by 100 ns of a production run in NVT for analysis of the equilibrium properties at a sampling interval of 10 ps. A cut off of $15 \AA$ was applied to account for the short-range electrostatic and van der Waals interactions. The particle mesh Ewald method ${ }^{42}$ was employed for the long-range electrostatics interaction. The LINCS algorithm ${ }^{43}$ was implemented to constrain the bond between H-atom and a bound heavy atom at its equilibrium length.

\section{Analysis Methods}

Construction of Intermolecular Networks of Interactions. Undirected, unweighted graphs (networks) were generated for each frame of the trajectory by considering each individual molecule as a vertex (or node), and an edge existing between nodes if certain distance criteria are satisfied. Graphs were generated from the 100 ns production trajectories at a sampling interval of 100 ps. The ChemNetworks ${ }^{22}$ software was employed for graph construction, as this accounts for periodic boundary conditions and contains several graph correction and analysis features (vide infra). Further analysis of the different graph-based descriptors were carried out using the NetworkX python package. ${ }^{44}$

Clusters (or aggregates) are defined as the components that are disconnected from the total network, where the composition of the cluster classifies the aggregate as either "homo-

geneous" (all nodes are the same type of molecule) or "heterogeneous" (nodes representing different molecular types). Note that some heterogeneous clusters can be composed of smaller homogeneous domains. Based on the system composition, three homogeneous aggregates 
can be formed, comprised solely of TODGA, water or nitric acid, and four heterogeneous aggregates may be formed, consisting of TODGA- $\mathrm{H}_{2} \mathrm{O}$, TODGA- $\mathrm{HNO}_{3}, \mathrm{H}_{2} \mathrm{O}-\mathrm{HNO}_{3}$ and TODGA- $\mathrm{H}_{2} \mathrm{O}-\mathrm{HNO}_{3}$. The cluster size is the total number of nodes within a component. Identifying the appropriate criterion for defining edges of interactions is a challenging task that requires significant consideration. A common practice is to obtain a distance-based cutoff based upon the radial distribution function (RDF) between appropriate particle pairs. ${ }^{45,46}$ In the case of amphiphiles like TODGA a weak dipole-dipole interaction drives association that can be manifested in different pair-wise particle-particle correlations. Stronger, directed interactions - as in hydrogen bonding - support more straightforward edge definitions. Further, it is important to recognize that a rigid geometric criterion can introduce artifacts into the graph structure due to thermal oscillations around the cutoff criterion and these features must be corrected for (vide infra).

TODGA-TODGA Edge Definitions. TODGA is a large molecule, with an end-to-end distance above $24 \AA$; cf. Figure S2. Several different geometric criterion were examined to define TODGA-TODGA interactions, based upon potential hydrophilic interactions mediated by the TODGA core ethereal and carbonyl O-atoms, and steric and hydrophobic interactions associated with the four $n$-octyl chains. Three different geometric cut-off criteria (labelled I-III) were examined for solution conditions C1 and C2 (Table S5, Supporting information) where there exists experimental data regarding the preferred TODGA oligomeric states. ${ }^{11,25,26}$ Although detailed comparisons are provided within the Supplementary Information, the best agreement with experiments for the geometric criterion was obtained for criterion III (cf. Figure S3) which imposes a distance cutoff between inter-TODGA etherealethereal O-atoms of $12 \AA$ and a minimum tail-tail proximity of $7.5 \AA$ (cf. Figure S2). A dynamics-based correction scheme proposed by Ozkanlar et al. ${ }^{47}$ was employed with these cut-off values to help remove additional artifacts created by rigid cutoff parameters (referred as criterion III-corr in Supporting Information).

Definitions of Hydrogen Bonding. Several different types of hydrogen bonding inter- 
actions are possible among the polar solutes within different system compositions under study, including: $\mathrm{H}_{2} \mathrm{O} \ldots \mathrm{H}_{2} \mathrm{O}, \mathrm{H}_{2} \mathrm{O} \ldots \mathrm{HNO}_{3}$ (water donating), $\mathrm{O}_{3} \mathrm{NH}_{\ldots} . . \mathrm{OH}_{2}$ (nitric acid donating). Amongst water and nitric acid, a distance cutoff of $2.5 \AA$ was set between donor(H)acceptor(O) for identifying hydrogen bond interactions. Further, for $\mathrm{HBs}$ among $\mathrm{H}_{2} \mathrm{O}$, an additional angle based cutoff criterion (less than $30^{\circ}$ for $\angle \mathrm{O}-\mathrm{H} \ldots \mathrm{O}$ ) was imposed along with the distance threshold. ${ }^{47}$ For HB interactions involving TODGA (i.e. $\mathrm{H}_{2} \mathrm{O}$...TODGA (water donating), $\mathrm{O}_{3} \mathrm{NH}$...TODGA (nitric acid donating)), a O...O distance cutoff of $3.8 \AA$ was employed based on the first minimum of corresponding RDF profiles (cf. Figure S4).

Local vs. Extended Aggregation. To learn more about the formation and growth of aggregates, as well as the changes to their the change network topology, two classes of clustering are delineated based upon the nature of the interactions that promote the aggregation process: 1) local aggregation and 2) extended aggregation. Local aggregation is characterized by a cluster that is dominated by TODGA nodes that have an edge irrespective of whether any $\mathrm{H}_{2} \mathrm{O}$ or $\mathrm{HNO}_{3}$ are present. In contrast, extended aggregation occurs when a homogeneous TODGA cluster is connected to a homogeneous water cluster but where each TODGA cluster is distance-separated (having no edge). In other words, in the extended aggregation, water clusters link otherwise separated TODGA cluster(s) and in this manner TODGA clusters are connected through non-local interactions. The same definitions are applicable to $\mathrm{HNO}_{3}$-containing cluster systems. Figure S5 illustrates the potential cluster compositions and their associated definitions.

Eccentricity Distribution Within Clusters. To evaluate the connectivity within the homo- and heterogeneous clusters, we computed the eccentricity distribution of all nodes using NetworkX. ${ }^{44}$ Within the formalism of graph theory, the eccentricity $\left(e_{v}\right)$ of a node is defined as the maximum graph-distance between the node with all other nodes present within the network.

$$
e_{v}=\max \{d(v, u), u \in v(G)\}
$$


here, $d(v, u)$ is the graph-distance between $u$ and $v$ node within graph $G$. The maximum eccentricity is the diameter of the graph. ${ }^{48}$ Thus, the distribution of this parameter with respect to all nodes reflects the span and connectivity of the network. Nodes with higher eccentricities indicate their participation within a cluster that has longer pathways, while a minimum eccentricity value of 1 arises when the node is directly connected to all the other nodes within the network (occurring only in small clusters). An illustration of eccentricity distributions for several different graph topologies is illustrated in Figure S6.

\section{Results and discussion}

In the absence of a polar solute, TODGA molecules interact via weak electrostatic dipoledipole forces. Experimentally, a dynamic equilibrium between a monomer and dimer is observed at a minimal concentration of $\mathrm{H}_{2} \mathrm{O}$ and acid. ${ }^{11,26}$ This behavior is reproduced within solution $\mathbf{A}$, where over $53 \%$ of all TODGA remain in a monomeric state, $23 \%$ in a dimer and $14 \%$ in a trimer. Moving forward, we now consider solution $\mathbf{B}$ conditions, so as to rigorously understand the role of water upon aggregation, followed by the $\mathbf{C}$ solutions where the perturbative role of $\mathrm{HNO}_{3}$ is identified.

\section{Concentration Dependent Aggregation Switching by Water}

Although only $9 \%$ of all TODGA participate in (TODGA) $)_{m}\left(\mathrm{H}_{2} \mathrm{O}\right)_{n}$ clusters with $m>3$ at $0.02 \mathrm{M} \mathrm{H}_{2} \mathrm{O}$, the growth of larger clusters is appreciable as water content is increased. At 0.05 $\mathrm{M} \mathrm{H}_{2} \mathrm{O}, 27 \%$ of TODGA reside in clusters with $m>3$ and at $0.2 \mathrm{M} \mathrm{H}_{2} \mathrm{O} 34 \%$ of TODGA exist in large clusters (Figure 1, Figure S7). These clusters are almost entirely heterogeneous in composition (TODGA $)_{m}\left(\mathrm{H}_{2} \mathrm{O}\right)_{n}$ and a significant decrease in the TODGA diffusion coefficient is observed (cf. Table S6, Supporting information). A non-linear, but positive, correlation is observed with respect to the water content within the heterogeneous clusters and the total cluster size (cf. Figure S8 and S9, Supporting information). Interestingly, 
the short-range TODGA-TODGA, TODGA-water and water-water nonbonded interaction energies (cf. Figure S10) as a function of $\left[\mathrm{H}_{2} \mathrm{O}\right]$ in the organic phase further indicate that the increase in water content has a noticeable indirect stabilizing effect on TODGA-TODGA interaction.

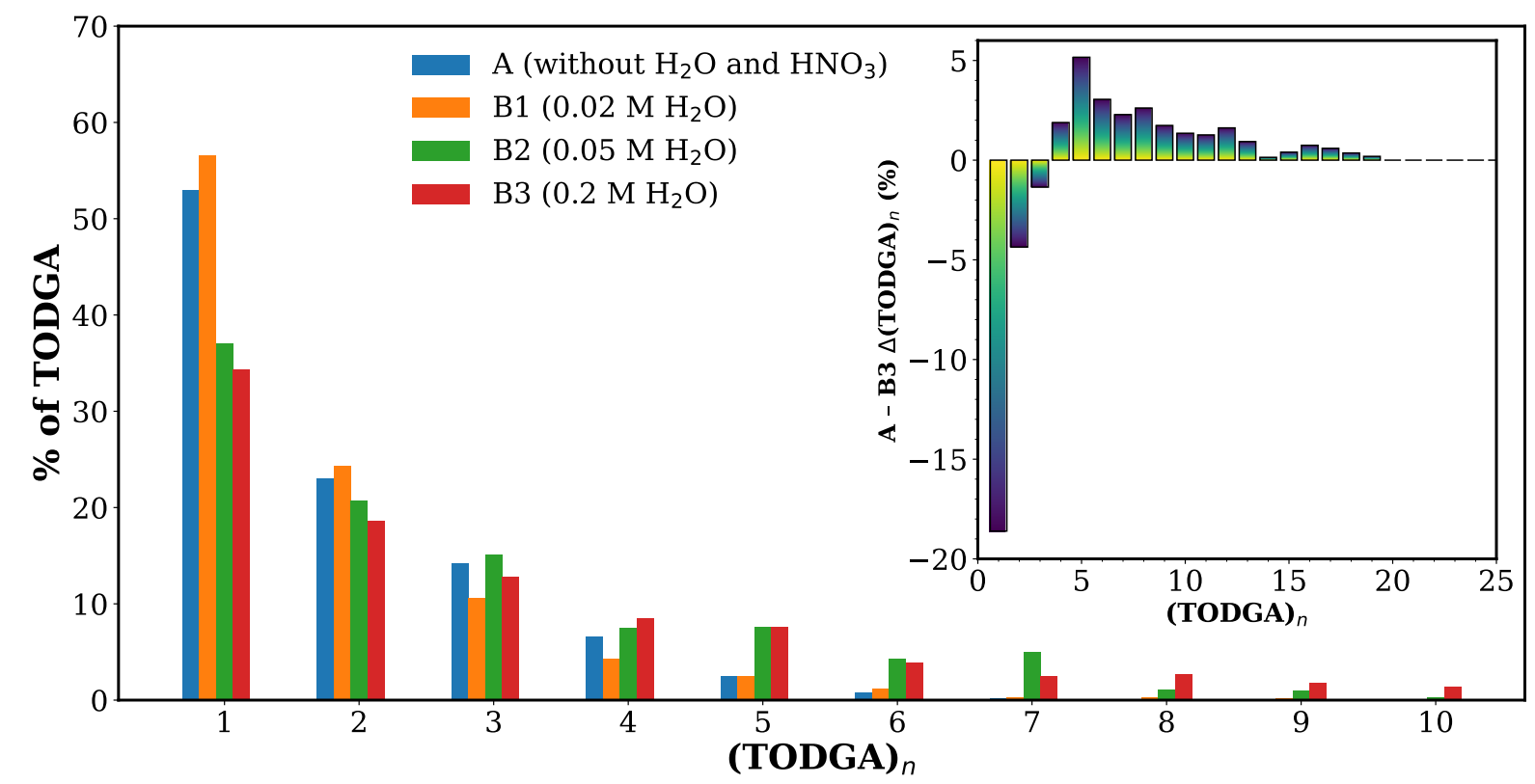

Figure 1: Cluster distribution with the increase of water concentration: Percentage of TODGA are plotted against the cluster size. The inset plot shows the relative change in the percentage of clusters against the cluster size from system A to B3.

To elucidate whether water is serving to enhance local or extended aggregation, we begin by studying the distribution of $\mathrm{H}_{2} \mathrm{O}$ in three different zones around individual TODGA molecules (cf. Table 2). The zones are defined based upon distances from the TODGA carbonyl and ethereal O-atoms using the around selection feature of the MDAnalysis toolkit. ${ }^{49}$ Zone 1 is defined by a distance $0-0.38 \mathrm{~nm}$, Zone $20.38-1 \mathrm{~nm}$ and Zone $3>1 \mathrm{~nm}$. These results indicate that with increasing water in the organic media, the population of $\mathrm{H}_{2} \mathrm{O}$ in Zone 2 (the $2^{\text {nd }}$ and $3^{\text {rd }}$ solvation shells of TODGA) increases more rapidly than in Zone 1 (the $1^{\text {st }}$ solvation shell). This complements the modest increase in hydrogen bonding between $\mathrm{H}_{2} \mathrm{O}$ and TODGA versus a much sharper $\mathrm{H}_{2} \mathrm{O} \ldots \mathrm{H}_{2} \mathrm{O}$ HB increase as we traverse $\mathbf{B} 1$ to $\mathbf{B} 2$ to $\mathbf{B} 3$ conditions (cf. Table 2). 
Table 2: (Top) Average number of hydrogen bonds among TODGA, $\mathrm{H}_{2} \mathrm{O}$ and $\mathrm{HNO}_{3}$. (Bottom) Percent distribution of water and nitric acid around TODGA O-atoms (out of all $\mathrm{H}_{2} \mathrm{O}$ or $\mathrm{HNO}_{3}$ present in the system). Three zones are defined based on the distance from the carbonyl and ethereal O-atoms; Zone 1: 0 - $0.38 \mathrm{~nm}$, Zone 2: $0.38-1 \mathrm{~nm}$, and Zone 3: >1 nm.

\begin{tabular}{|c|c|c|c|c|c|c|}
\hline \multirow{2}{*}{ Conditions } & \multicolumn{6}{|c|}{ Hydrogen Bonds } \\
\hline & \multicolumn{2}{|c|}{$\mathrm{H}_{2} \mathrm{O} \ldots \mathrm{H}_{2} \mathrm{O}^{a}$} & \multicolumn{3}{|c|}{ TODGA $\ldots \mathrm{H}_{2} \mathrm{O}^{b} \quad \mathrm{H}_{2} \mathrm{O} \ldots \mathrm{H}$} & $\mathrm{TODGA} . . \mathrm{HNO}_{3}^{b}$ \\
\hline B1 & \multicolumn{2}{|c|}{0.21} & 0.14 & \multicolumn{2}{|c|}{-} & - \\
\hline B2 & \multicolumn{2}{|c|}{0.47} & 0.33 & \multicolumn{2}{|c|}{-} & - \\
\hline B3 & \multicolumn{2}{|c|}{1.22} & 0.46 & \multicolumn{2}{|c|}{-} & - \\
\hline C3 & \multicolumn{2}{|c|}{1.12} & 0.58 & \multicolumn{2}{|c|}{0.06} & 0.07 \\
\hline $\mathrm{C} 4$ & \multicolumn{2}{|c|}{1.02} & 1.02 & \multicolumn{2}{|c|}{0.24} & 0.21 \\
\hline C3 & \multicolumn{2}{|c|}{0.69} & 1.33 & \multicolumn{2}{|c|}{0.70} & 0.28 \\
\hline \multirow{2}{*}{ Conditions } & \multicolumn{3}{|c|}{ Percent of Water } & \multicolumn{3}{|c|}{ Percent of Nitric Acid } \\
\hline & Zone 1 & Zone 2 & Zone 3 & Zone 1 & Zone 2 & Zone 3 \\
\hline B1 & 77.098 & 17.732 & 5.170 & - & - & - \\
\hline B2 & 68.528 & 29.104 & 2.368 & - & - & - \\
\hline B3 & 38.836 & 59.561 & 1.603 & - & - & - \\
\hline C3 & 42.995 & 52.776 & 4.229 & 19.863 & 79.387 & 0.749 \\
\hline $\mathrm{C} 4$ & 53.023 & 45.797 & 1.180 & 50.160 & 48.748 & 1.092 \\
\hline C5 & 62.230 & 35.533 & 2.237 & 42.605 & 54.486 & 2.909 \\
\hline
\end{tabular}

${ }^{a}$ Values indicate the average number of hydrogen bonds per $\mathrm{H}_{2} \mathrm{O} .{ }^{b}$ Values correspond to the average number of hydrogen bonds per TODGA. 
The propensity for two different cluster configurations was then examined:

1. TODGA clusters that are solvated by separated water clusters (labelled "water-extractantwater" or "W-E-W"). In this type of arrangements TODGA molecules have direct interactions with each other and are connected components of the cluster subgraph. Thus W-E-W is considered local aggregation because of the locality of the TODGATODGA interactions.

2. Water clusters that link separated TODGA clusters (labelled "extractant-water-extractant" or "E-W-E"). These instances represent extended aggregation where TODGA interact through the HB network of water.

The distribution of E-W-E and W-E-W heterogeneous clusters is presented in Figure 2, as represented by the formation matrix whose rows and columns are the size of the terminal homogeneous sub-clusters while the inset presents the size distribution of the central (bridging) sub-cluster. In general, there is a predominance of W-E-W cluster configurations however the frequency of E-W-E increases with increasing water concentration (cf. Figure S11, Supporting Information). Smaller $\left(\mathrm{H}_{2} \mathrm{O}\right)_{n}$ clusters are correlated with the W-E-W cluster configurations, whereas large water clusters are correlated with the E-W-E. As the total water content is increased the relative percent of E-W-E clusters increases from $0.6 \%$ at $0.02 \mathrm{M} \mathrm{H}_{2} \mathrm{O}$ (B1) to $2.0 \%$ at $0.05 \mathrm{M} \mathrm{H}_{2} \mathrm{O}$ (B2) to $18.5 \%$ at $0.2 \mathrm{M} \mathrm{H}_{2} \mathrm{O}$ (B3).

The topological properties of the network of intermolecular interactions within the E-WE and W-E-W clusters was examined via the eccentricity distribution of the molecular nodes (Eqn. 1), as shown in Figure 3. As described in the computational section, the eccentricity of a node is the maximum graph-distance between the node with all other nodes present within the graph-network. Being a node-specific property, the distribution of eccentricities reflects the internal connectivity as well as the span of network. For TODGA-water mixedaggregates, the shift towards higher eccentricities is substantial as we traverse from low concentration to high water concentration. This would be anticipated for the growth of the 


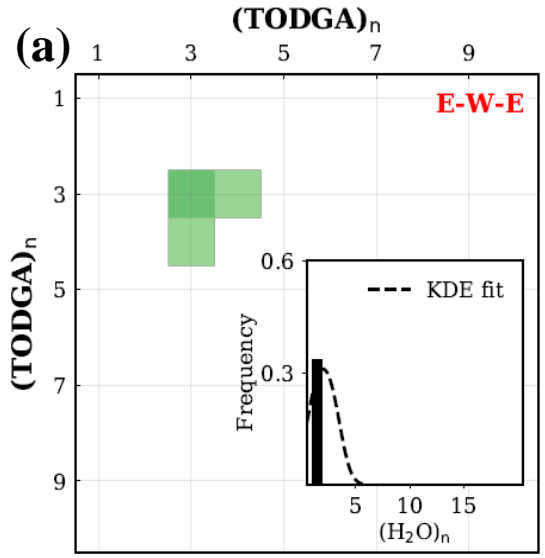

(TODGA)

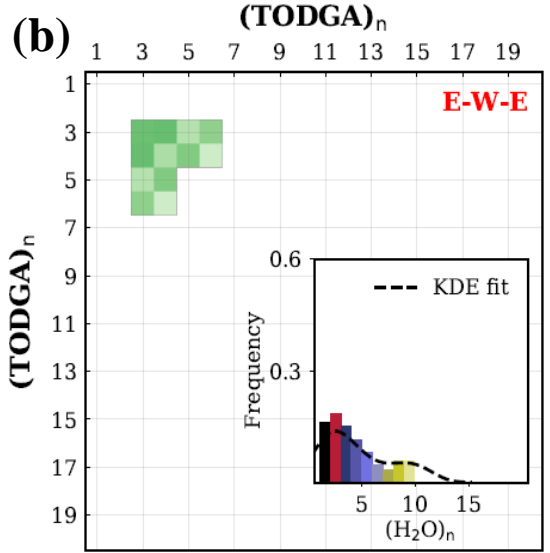

(TODGA)

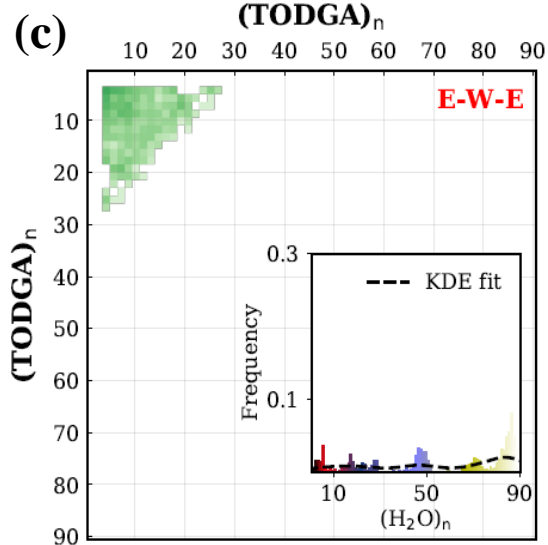

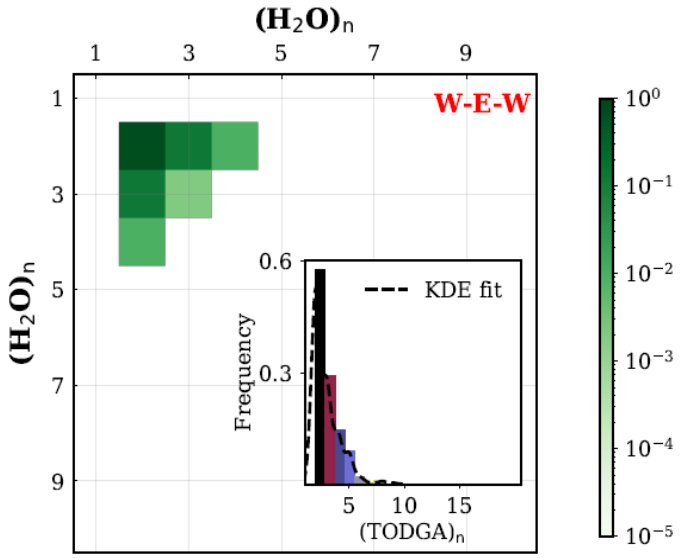

$\left(\mathbf{H}_{2} \mathbf{O}\right)_{n}$

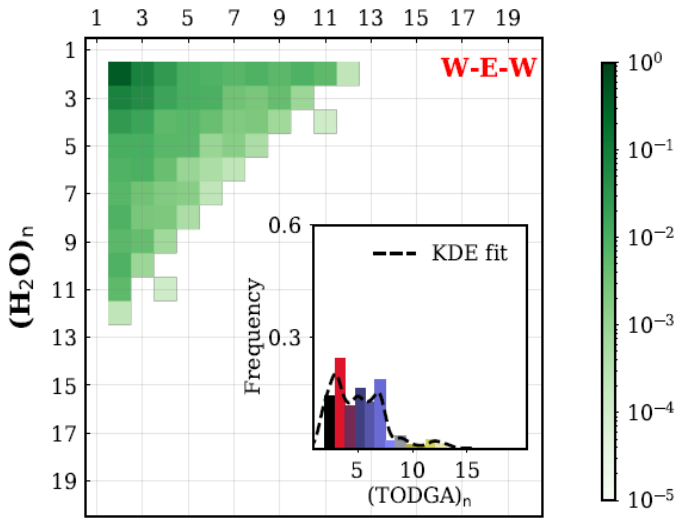

$\left(\mathbf{H}_{2} \mathbf{O}\right)_{n}$

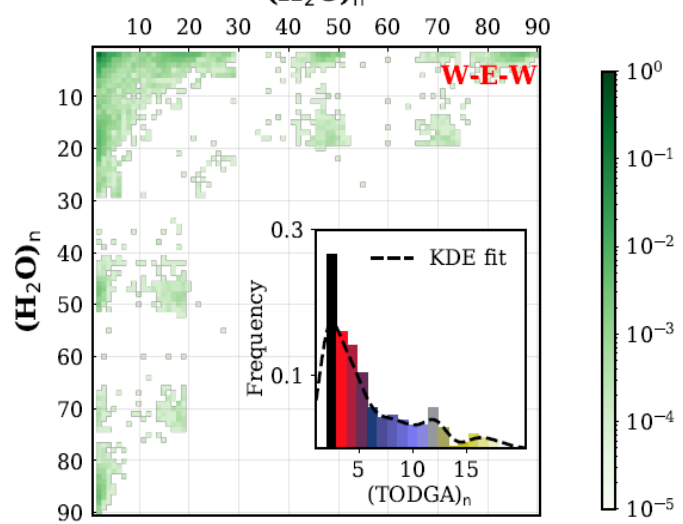

Figure 2: The growth of Extractant-Water-Extractant (E-W-E) mixed-aggregates where water bridges the TODGA clusters and Water-Extractant-Water (W-E-W) mixed-aggregates where TODGA bridges the water clusters for (a) B1 (top), B2 (middle) and B3 (bottom) solution conditions. The color bar is in logarithmic scale and indicate the normalized proportion of the total occurrences of E-W-E and W-E-W mixed-aggregates as function of the cluster sizes. The inset box within the plots shows the normalized frequency distribution of occurrences ( $\mathrm{Y}$ axis) of the bridging constituents (left panel: water; right panel: TODGA) with respect to their cluster size ( $\mathrm{X}$ axis). The shape of the distribution in the inset box is obtained using gaussian kernel density estimate (KDE). 
water cluster hydrogen bond network and its interactions with the TODGA clusters that it links together. In combination, these data support a role for water of acting as driving force behind local aggregation at low water content within the organic phase and that as the water content increases, competition emerges for the preferential solvation of $\mathrm{H}_{2} \mathrm{O}$ with itself over solvation of TODGA. When larger water clusters are formed, they enable extended aggregation behavior within the solution where the total cluster size is further significantly larger than with the W-E-W configuration (Figure S12, Supporting information).
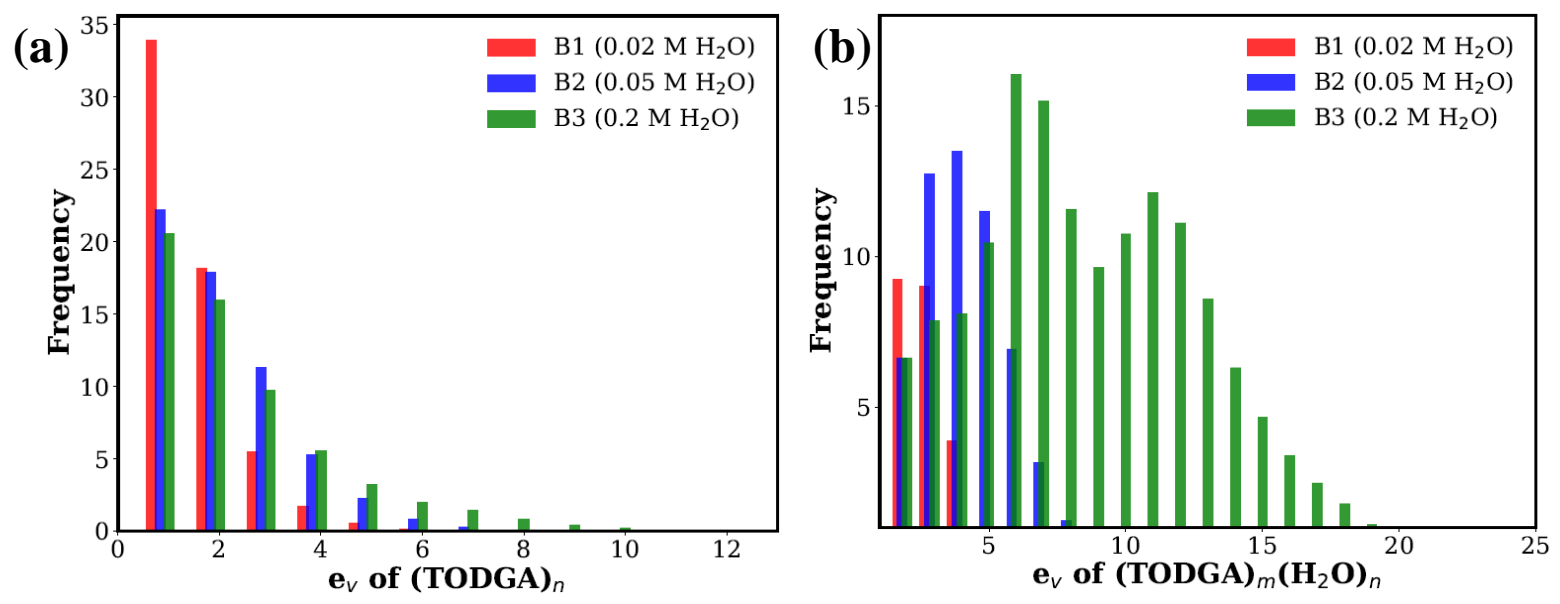

Figure 3: a) Distribution of eccentricities $\left(\mathrm{e}_{v}\right)$ associated with the TODGA clusters for B1, B2 and B3 system. b) Distribution of $\left(\mathrm{e}_{v}\right)$ associated with the TODGA-Water network for $\mathrm{B} 1, \mathrm{~B} 2$ and B3 system. Frequency quantifies the total number of instances of $\mathrm{e}_{v}$ for all the molecules of associated aggregate and mized-aggregate type.

\section{Nitric Acid Inhibits Extended Aggregation}

Prior experimental study ${ }^{10,25,50}$ has reported that an increase in $\left[\mathrm{HNO}_{3}\right]_{a q}$ leads to growth of aggregate size within the organic phase. An increase in the stickness parameter required to fit SAXS data ${ }^{50}$ also supports enhanced inter-aggregate interaction at higher aqueous acidities. However, it is difficult to interpret whether such effects derive solely from an increased $\left[\mathrm{HNO}_{3}\right]_{\text {org }}$, as extracted acid may potentially form protonated solvates, for example of the form "extractant... $\left(\mathrm{HNO}_{3}\right)_{x}$ ". Other hypotheses could easily be generated because as the $\left[\mathrm{HNO}_{3}\right]_{\text {org }}$ increases - so too does $\left[\mathrm{H}_{2} \mathrm{O}\right]_{\text {org }}$. This introduces competitive or synergistic 
interactions as a result of the differing hydrogen bond capabilities of these two polar solutes.

The B3 solution conditions $\left(0.2 \mathrm{M} \mathrm{H}_{2} \mathrm{O}\right)$ was used as the basis for investigating the impact of $\mathrm{HNO}_{3}$ on the aggregation of TODGA, where the acid concentration is systematically increased from 0.01 M (forming the C3 solution) to 0.05 M (C4) to 0.15 M (C5). Perhaps unsurprisingly, the single $\mathrm{HB}$ donor site of $\mathrm{HNO}_{3}$ limits the ability of nitric acid form homogeneous aggregates and over $90 \%$ of $\mathrm{HNO}_{3}$ are observed to remain in a monomeric form (with no $\mathrm{HB}$ to other $\mathrm{HNO}_{3}$ ). The molecular form of nitric acid has three $\mathrm{HB}$ acceptor sites and one donor site, which introduce significant competition for hydrogen bonding amongst the two $\mathrm{HB}$ acceptor and donor sites of $\mathrm{H}_{2} \mathrm{O}$. Analysis of the pair-wise interaction energies suggest an increasing stabilization of the $\mathrm{HNO}_{3} \ldots \mathrm{H}_{2} \mathrm{O}$ interaction at the expense of destabilizing $\mathrm{H}_{2} \mathrm{O} \ldots \mathrm{H}_{2} \mathrm{O}$ interactions as $\left[\mathrm{HNO}_{3}\right]_{\text {org }}$ increases (cf. Figure S13). As shown in Figure S14, introduction of $\mathrm{HNO}_{3}$ also significantly perturbs the water clustering process where hydrogen bonding of $\mathrm{H}_{2} \mathrm{O}$ and $\mathrm{HNO}_{3}$ restricts the formation of water clusters over the size of c.a. 30 within the $\mathbf{C 5}$ solution. This is well-supported by the subsequent increase in solvent accessible surface area (SASA) per water molecule upon increasing acid concentration (Table S7, Supporting information). In-depth analysis on the inter-connectivity of the $\mathrm{HNO}_{3} \ldots \mathrm{H}_{2} \mathrm{O} \mathrm{HB}$ network suggests that with increasing concentration, $\mathrm{HNO}_{3}$ participates in forming mixed aggregates of the form $\mathrm{N}-\mathrm{W}-\mathrm{N}$ (nitric acid-water-nitric acid) and $\mathrm{W}-\mathrm{N}-\mathrm{W}$ (water-nitric acid-water) with substantial preference for former (cf. Figure S15 and S16, Supporting Information). As a result, the presence of $\mathrm{HNO}_{3}$ reduces the hydrogen bond network connectivity among $\mathrm{H}_{2} \mathrm{O}$ and shortens the associated network length. The eccentricity distributions of the hydrogen bond network of water clusters reflects a gradual reduction of graph distance between $\mathrm{H}_{2} \mathrm{O}$ with increasing $\left[\mathrm{HNO}_{3}\right]$. Concomitantly, there is a decreasing trend of hydrogen bonds between $\mathrm{H}_{2} \mathrm{O}$ and an increase in the propensity of $\mathrm{H}_{2} \mathrm{O}$ to solvate more TODGA molecules (growth in the average number of HBs between TODGA and $\mathrm{H}_{2} \mathrm{O}$ shown in Table 2).

These data complement the observed changes to distribution of $\mathrm{H}_{2} \mathrm{O}$ and $\mathrm{HNO}_{3}$ around 
TODGA in various zones as $\left[\mathrm{HNO}_{3}\right]$ is increased. Specifically, the percentage of $\mathrm{H}_{2} \mathrm{O}$ around TODGA in its $1^{\text {st }}$ solvation shell (Zone 1) increases whereas less waters partition within Zone 2 (cf. Table 2). At low $\left[\mathrm{HNO}_{3}\right]\left(0.01 \mathrm{M}\right.$; C3), $>80 \%$ of $\mathrm{HNO}_{3}$ remain in the Zone 2 while a significantly higher percentage migrate into the $1^{\text {st }}$ solvation shell of TODGA as the total nitric acid concentration is increased. Thus, there are enhanced interactions between both polar solutes and the hydrophilic core of TODGA as acid is added to the solution. The disruption in the water HB network by nitric acid has a direct impact over the cluster size distribution of TODGA and on the TODGA-water mixed-aggregates (Figure S17 and S18, Supporting information). Specifically, reducing the size of water clusters severely inhibits the formation of TODGA clusters that are linked by those water clusters as shown in Figure S14 (Supporting information). The size of the TODGA-water and TODGA-water-nitric acid mixed aggregates (cf. Figure 5) remains restricted due to the smaller core of water-nitric acid mixed-aggregates. Further, contrary to the case of water without acid, there is a decrease in the formation of E-W-E type of mixed-aggregates with increasing acid concentration (Figure 4 and Figure S11). The distribution of eccentricities of W-E-W and E-W-E aggregates also demonstrates a reduction of network length, suggesting that in the presence of water, nitric acid reduces the inter-connectivity among the mixed-aggregates (cf. Figure 6).

These predicted trends are somewhat counter-intuitive to the implied role of nitric acid within the experimental literature. Although, it has been perceived in several experiments ${ }^{10,25,50}$ that increase in $\left[\mathrm{HNO}_{3}\right]_{a q}$ leads to better amphiphile aggregation, it is important to emphasize that the aqueous acidity increases both the concentration of $\left[\mathrm{H}_{2} \mathrm{O}\right]_{\text {org }}$ and $\left[\mathrm{HNO}_{3}\right]_{\text {org }} .{ }^{16}$ This work demonstrates that $\mathrm{H}_{2} \mathrm{O}$ supports both local and extended aggregation as its concentration in the organic phase increases, while the competitive hydrogen bond interactions with nitric acid decrease extended aggregation. Thus, we propose that the increase in the size of amphiphile aggregates observed in prior experiments likely derives from the enhanced concentration of water (caused by coextraction with $\mathrm{HNO}_{3}$ ) rather than the role of the acid upon self-assembly. Undoubtedly, the concentration of water in the organic 
phase is crucial to tune the degree of local and extended aggregation events involving nitric acid. A comparison of the $\mathbf{B} \mathbf{1}$ and $\mathbf{C} 1$ systems alongside the $\mathbf{B} 2$ and $\mathbf{C} 2$ systems provide further intuition about the dependency between nitric acid concentration and the extent of aggregation. The small quantity of acid that present in $\mathbf{C} 1(0.02 \mathrm{M}$ water and $0.01 \mathrm{M}$ acid) relative to B1 (0.02 M water) does not appreciably perturb the small existing water network. The dispersed $\mathrm{H}_{2} \mathrm{O}$ and $\mathrm{HNO}_{3}$ solvate TODGA molecules without little interaction amongst themselves. Indeed the size of TODGA clusters from $\mathbf{B} \mathbf{1}$ to $\mathbf{C} \mathbf{1}$ is increased by the individual bridging of the isolated polar solutes (cf. Figure S19, Supporting Information). Increasing the water and acid content to (i.e. C2 $(0.05 \mathrm{M}$ water and $0.05 \mathrm{M}$ acid); relative to B2 ( $0.05 \mathrm{M}$ water) creates competition between $\mathrm{H}_{2} \mathrm{O} \ldots \mathrm{H}_{2} \mathrm{O}$ hydrogen bonding and $\mathrm{H}_{2} \mathrm{O} \ldots \mathrm{HNO}_{3}$, and instead of the growth of large water clusters observed in the absence of nitric acid, the relative size of the TODGA clusters is restricted (cf. Figure S19, Supporting Information). 

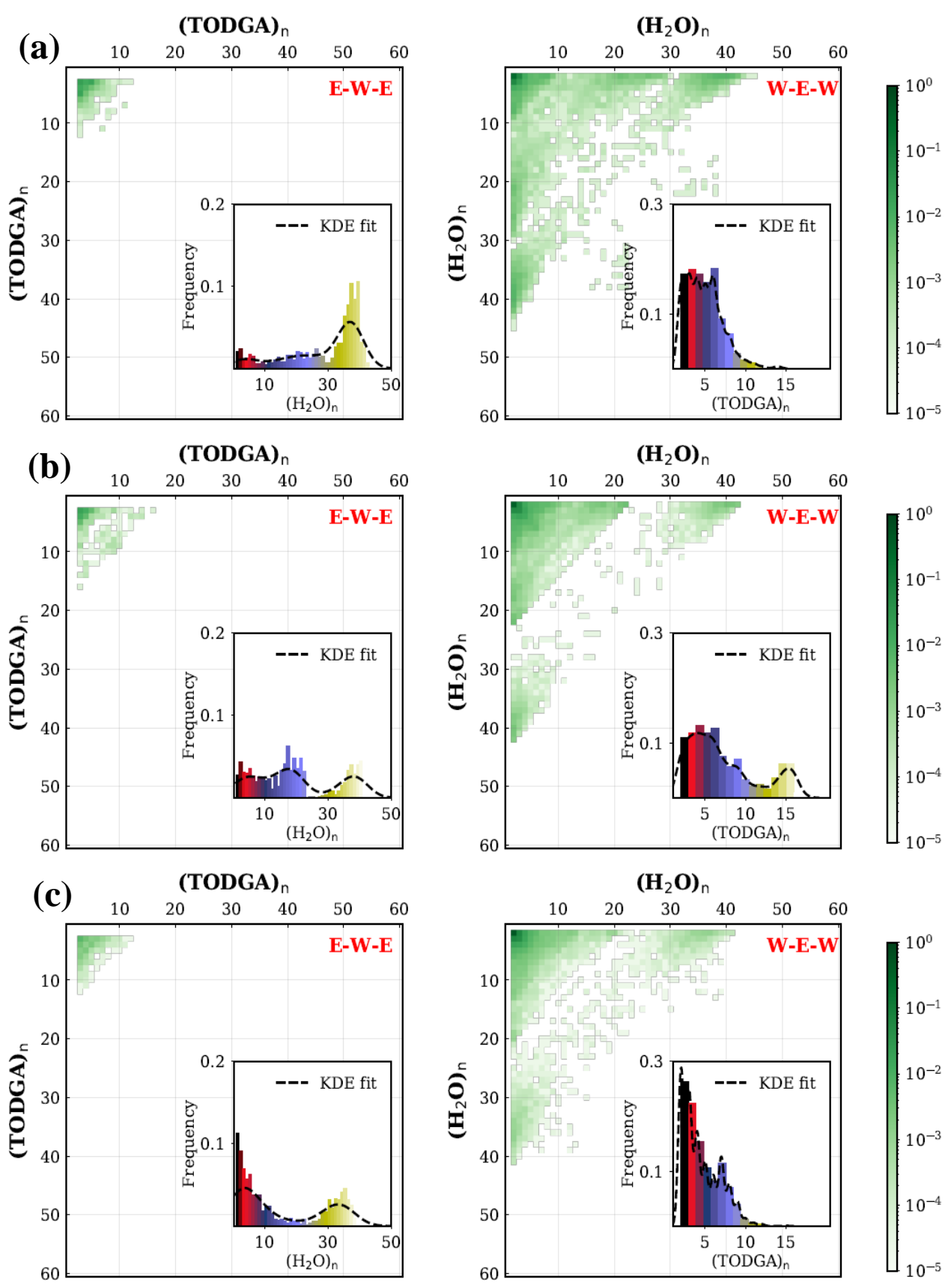

Figure 4: The plot describes the growth of bridged assemblies between water and TODGA for C3 (top), C4 (middle) and C5 (bottom) systems. Left panel represents occurrences of Extractant-Water-Extractant (E-W-E) mixed-aggregates where water bridges the TODGA clusters. Right panel represents occurrences of Water-Extractant-Water (W-E-W) mixedaggregates where TODGA bridges the water clusters. The color bar is in logarithmic scale and indicate the normalized proportion of the total occurrences of E-W-E and W-E-W mixed-aggregates as function of the cluster sizes of bridged entities. The inset box within the plots shows the normalized frequency distribution of occurrences (Y axis) of the bridging constituents (left panel: water; right panel: TODGA) with respect to their cluster size (X axis).The shape of the distribution in the inset box is obtained using gaussian kernel density estimate (KDE). 

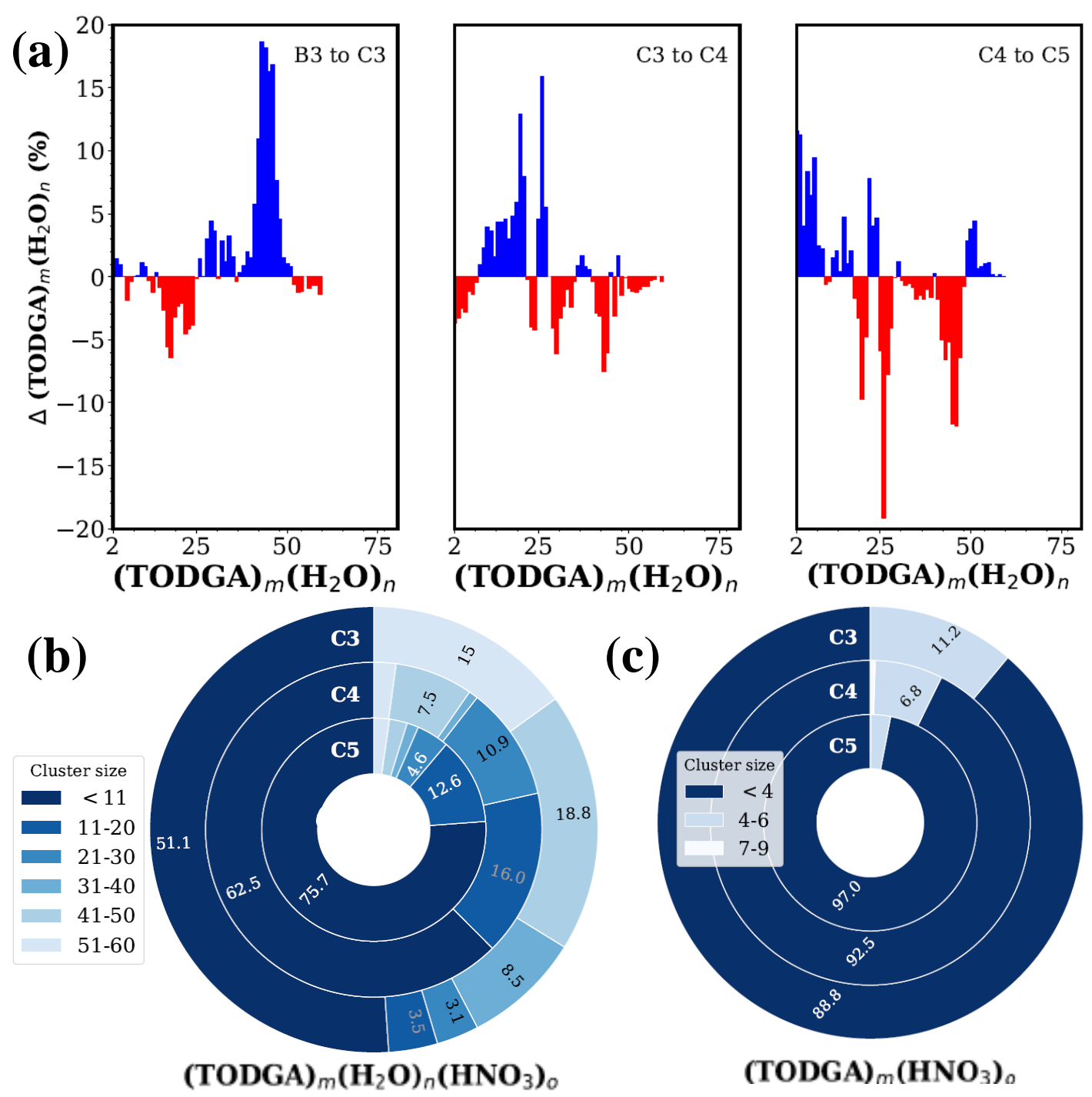

Figure 5: (a) The relative change in the percentage of (TODGA $)_{m}\left(\mathrm{H}_{2} \mathrm{O}\right)_{n}$ mixed-aggregates progressively from B3 to C3, C4 and C5 systems are plotted against the cluster size $(\mathrm{m}+\mathrm{n})$. Radial bar chart on cluster distribution of (b) $(\mathrm{TODGA})_{m}\left(\mathrm{H}_{2} \mathrm{O}\right)_{n}\left(\mathrm{HNO}_{3}\right)_{o}$ and (c) (TODGA $)_{m}\left(\mathrm{HNO}_{3}\right)_{o}$ mixed aggregates under $\mathrm{C} 3, \mathrm{C} 4$ and $\mathrm{C} 5$ condition (values on the chart indicates $\%$ of clusters within respective size interval (only values $>3 \%$ are shown for clarity). 

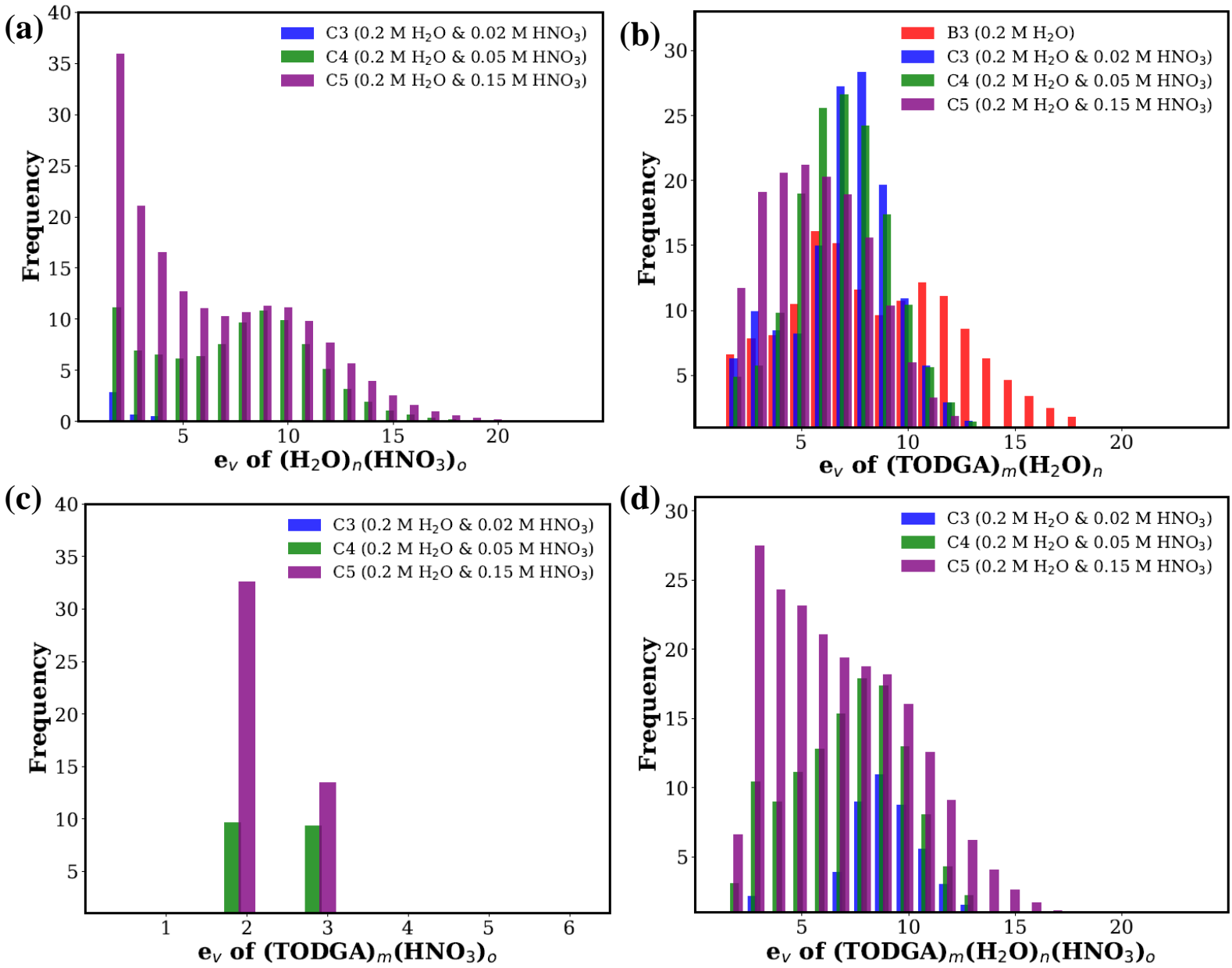

Figure 6: Distribution of eccentricities $\left(\mathrm{e}_{v}\right)$ associated with (a) Water-Nitric acid network, (b) TODGA-Water network, (c) TODGA-Nitric acid (d) TODGA-Water-Nitric acid network under $\mathrm{C} 3, \mathrm{C} 4$ and $\mathrm{C} 5$ conditions. Frequency quantifies the total number of instances of $\mathrm{e}_{v}$ for all the molecules of associated mixed-aggregates type. 


\section{Conclusion}

The presence of water and acid in non-polar media has been previously observed to enhance self-assembly of amphiphiles, however mechanistic insight has been absent, largely due to the complexity of partitioning the individual role of these polar solutes as a function of concentration. Although a synergistic effect could be responsible for enhanced amphiphile aggregation, the varying hydrogen bond characteristics of water and acids, combined with different preferred solvation environments, could also allow for concentration dependent competition. Within this vein, the current work leverages a liquid-liquid extraction system as a platform for exploring the self-assembly of the amphiphile TODGA in the presence of water and nitric acid in dodecane. Detailed molecular dynamics simulations combined with graph-based cluster and network analyses reveal disparate roles of $\mathrm{H}_{2} \mathrm{O}$ and $\mathrm{HNO}_{3}$ - where water has the dual ability to enhance local interactions of TODGA up to a certain concentration limit, where then water-water preferred solvation dominates and the formation of large water clusters serve to link TODGA clusters that assemble on the water surface periphery. The large number of accepting hydrogen bond sites in $\mathrm{HNO}_{3}$ compete with water-water hydrogen bonding when nitric acid is introduced into the organic solution. Nitric acid prevents the formation of large water clusters and thus limits the formation of extended TODGA aggregation. This tunes the clustering of TODGA and the controls formation of mixed-aggregates. As a consequence, unlike water, nitric acid is found to reduce the inter-connectivity of formed aggregates and allows growth of small and medium-sized mixed-aggregates. Despite the prevalent assumption within the experimental literature that nitric acid is generally responsible for the growth of TODGA assemblies when water and acid are extracted into TODGA laden organic phase, this work instead proposes that it is the role of water that predominates the observed aggregation phenomena. This work forms the basis for a fundamental understanding of how varying hydrogen bond characteristics and solvation properties influence self-assembly of amphiphiles in non-polar media. Further, as it pertains to LLE, the results from this work will help within the experimental design of 
separations systems that can tune aggregation behavior to influence separations efficiency.

\section{References}

(1) Herbst, F.; Schroter, K.; Gunkel, I.; Grøger, S.; Thurn-Albrecht, T.; Balbach, J.; Binder, W. H. Aggregation and chain dynamics in supramolecular polymers by dynamic rheology: cluster formation and self-aggregation. Macromolecules 2010, 43, 1000610016.

(2) Park, S.; Lim, J.-H.; Chung, S.-W.; Mirkin, C. A. Self-assembly of mesoscopic metalpolymer amphiphiles. Science 2004, 303, 348-351.

(3) Charleux, B.; Delaittre, G.; Rieger, J.; D’Agosto, F. Polymerization-induced selfassembly: from soluble macromolecules to block copolymer nano-objects in one step. Macromolecules 2012, 45, 6753-6765.

(4) Le Bon, C.; Nicolai, T.; Durand, D. Kinetics of aggregation and gelation of globular proteins after heat-induced denaturation. Macromolecules 1999, 32, 6120-6127.

(5) Yamada, Y. M.; Sarkar, S. M.; Uozumi, Y. Amphiphilic self-assembled polymeric copper catalyst to parts per million levels: click chemistry. Journal of the American Chemical Society 2012, 134, 9285-9290.

(6) Rösler, A.; Vandermeulen, G. W.; Klok, H.-A. Advanced drug delivery devices via selfassembly of amphiphilic block copolymers. Advanced drug delivery reviews 2012, 64, 270-279.

(7) Xu, X.-F.; Pan, C.-Y.; Zhang, W.-J.; Hong, C.-Y. Polymerization-induced self-assembly generating vesicles with adjustable $\mathrm{pH}$-responsive release performance. Macromolecules 2019, 52, 1965-1975. 
(8) Ianiro, A.; Wu, H.; van Rijt, M. M.; Vena, M. P.; Keizer, A. D.; Esteves, A. C. C.; Tuinier, R.; Friedrich, H.; Sommerdijk, N. A.; Patterson, J. P. Liquid-liquid phase separation during amphiphilic self-assembly. Nature chemistry 2019, 11, 320-328.

(9) Zemb, T.; Duvail, M.; Dufrêche, J.-F. Reverse Aggregates as Adaptive Self-Assembled Systems for Selective Liquid-Liquid Cation Extraction. Israel Journal of Chemistry 2013, 53, 108-112.

(10) Rama Swami, K.; Venkatesan, K.; Antony, M. Aggregation behavior of Alkyldiglycolamides in n-dodecane medium during the extraction of $\mathrm{Nd}$ (III) and nitric acid. Industrial \& Engineering Chemistry Research 2018, 57, 13490-13497.

(11) Yaita, T.; Herlinger, A.; Thiyagarajan, P.; Jensen, M. Influence of extractant aggregation on the extraction of trivalent f-element cations by a tetraalkyldiglycolamide. Solvent extraction and ion exchange 2004, 22, 553-571.

(12) s̃padina, M.; Bohinc, K.; Zemb, T.; Dufrêche, J.-F. Synergistic Solvent Extraction Is Driven by Entropy. ACS nano 2019, 13, 13745-13758.

(13) Ellis, R. J.; Meridiano, Y.; Muller, J.; Berthon, L.; Guilbaud, P.; Zorz, N.; Antonio, M. R.; Demars, T.; Zemb, T. Complexation-induced supramolecular assembly drives metal-ion extraction. Chemistry-A European Journal 2014, 20, 12796-12807.

(14) Campbell, E. L.; Holfeltz, V. E.; Hall, G. B.; Nash, K. L.; Lumetta, G. J.; Levitskaia, T. G. Nitric acid and water extraction by T2EHDGA in n-dodecane. Solvent Extraction and Ion Exchange 2017, 35, 586-603.

(15) Shamay, E. S.; Buch, V.; Parrinello, M.; Richmond, G. L. At the water's edge: Nitric acid as a weak acid. Journal of the American Chemical Society 2007, 129, 12910-12911.

(16) Gaillard, C.; Mazan, V.; Georg, S.; Klimchuk, O.; Sypula, M.; Billard, I.; Schurhammer, R.; Wipff, G. Acid extraction to a hydrophobic ionic liquid: the role of added 
tributylphosphate investigated by experiments and simulations. Physical Chemistry Chemical Physics 2012, 14, 5187-5199.

(17) Naito, K.; Suzuki, T. The mechanism of the extraction of several proton acids by trin-butyl phosphate. The Journal of Physical Chemistry 1962, 66, 983-988.

(18) Jiang, J.; Li, W.; Gao, H.; Wu, J. Extraction of inorganic acids with neutral phosphorus extractants based on a reverse micelle/microemulsion mechanism. Journal of colloid and interface science 2003, 268, 208-214.

(19) Ferru, G.; Gomes Rodrigues, D.; Berthon, L.; Diat, O.; Bauduin, P.; Guilbaud, P. Elucidation of the Structure of Organic Solutions in Solvent Extraction by Combining Molecular Dynamics and X-ray Scattering. Angewandte Chemie International Edition 2014, 53, 5346-5350.

(20) Motokawa, R.; Kobayashi, T.; Endo, H.; Mu, J.; Williams, C. D.; Masters, A. J.; Antonio, M. R.; Heller, W. T.; Nagao, M. A Telescoping View of Solute Architectures in a Complex Fluid System. ACS central science 2018, 5, 85-96.

(21) Servis, M. J.; Wu, D. T.; Braley, J. C. Network analysis and percolation transition in hydrogen bonded clusters: nitric acid and water extracted by tributyl phosphate. Physical Chemistry Chemical Physics 2017, 19, 11326-11339.

(22) Ozkanlar, A.; Clark, A. E. ChemNetworks: a complex network analysis tool for chemical systems. Journal of computational chemistry 2014, 35, 495-505.

(23) Ansari, S.; Pathak, P.; Manchanda, V.; Husain, M.; Prasad, A.; Parmar, V. N,N,N' $\mathrm{N}^{\prime}-$ tetraoctyl diglycolamide (TODGA): a promising extractant for actinide-partitioning from high-level waste (HLW). Solvent extraction and ion exchange 2005, 23, 463-479.

(24) Whittaker, D.; Geist, A.; Modolo, G.; Taylor, R.; Sarsfield, M.; Wilden, A. Applications 
of diglycolamide based solvent extraction processes in spent nuclear fuel reprocessing, part 1: TODGA. Solvent Extraction and Ion Exchange 2018, 36, 223-256.

(25) Nave, S.; Modolo, G.; Madic, C.; Testard, F. Aggregation properties of N,N,N', $\mathrm{N}^{\prime}-$ tetraoctyl-3-oxapentanediamide (TODGA) in n-dodecane. Solvent extraction and ion exchange 2004, 22, 527-551.

(26) Jensen, M. P.; Yaita, T.; Chiarizia, R. Reverse-micelle formation in the partitioning of trivalent f-element cations by biphasic systems containing a tetraalkyldiglycolamide. Langmuir 2007, 23, 4765-4774.

(27) Vo, Q. N.; Dang, L. X.; Nilsson, M.; Nguyen, H. D. Quantifying dimer and trimer formation by tri-n-butyl phosphates in n-dodecane: Molecular dynamics simulations. The Journal of Physical Chemistry B 2016, 120, 6985-6994.

(28) Wang, J.; Wolf, R. M.; Caldwell, J. W.; Kollman, P. A.; Case, D. A. Development and testing of a general amber force field. Journal of computational chemistry 2004, 25, $1157-1174$.

(29) Becke, A. D. Density-functional thermochemistry. I. The effect of the exchange-only gradient correction. The Journal of chemical physics 1992, 96, 2155-2160.

(30) Lee, C.; Yang, W.; Parr, R. G. Development of the Colle-Salvetti correlation-energy formula into a functional of the electron density. Physical review B 1988, 37, 785.

(31) Hehre, W. J.; Ditchfield, R.; Pople, J. A. Self - consistent molecular orbital methods. XII. Further extensions of Gaussian - type basis sets for use in molecular orbital studies of organic molecules. The Journal of Chemical Physics 1972, 56, 2257-2261.

(32) Hirata, M.; Guilbaud, P.; Dobler, M.; Tachimori, S. Molecular dynamics simulations for the complexation of Ln $3+$ and UO $22+$ ions with tridentate ligand diglycolamide (DGA). Physical Chemistry Chemical Physics 2003, 5, 691-695. 
(33) Ansari, S.; Mohapatra, P.; Prabhu, D.; Manchanda, V. Evaluation of N, N, N', N'tetraoctyl-3-oxapentane-diamide (TODGA) as a mobile carrier in remediation of nuclear waste using supported liquid membrane. Journal of membrane science 2007, 298, $169-174$.

(34) Ganguly, R.; Sharma, J. N.; Choudhury, N. TODGA based w/o microemulsion in dodecane: An insight into the micellar aggregation characteristics by dynamic light scattering and viscometry. Journal of colloid and interface science 2011, 355, 458-463.

(35) Jorgensen, W. L.; Jenson, C. Temperature dependence of TIP3P, SPC, and TIP4P water from NPT Monte Carlo simulations: Seeking temperatures of maximum density. Journal of computational chemistry 1998, 19, 1179-1186.

(36) Kuo, M. H.; David, A.; Kamelamela, N.; White, M.; Shultz, M. J. Nitric Acid- Water Interaction Probed via Isolation in Carbon Tetrachloride. The Journal of Physical Chemistry C 2007, 111, 8827-8831.

(37) Miller, Y.; Gerber, R. B. Dynamics of proton recombination with NO 3- anion in water clusters. physical chemistry chemical physics 2008, 10, 1091-1093.

(38) Martínez, L.; Andrade, R.; Birgin, E. G.; Martínez, J. M. PACKMOL: a package for building initial configurations for molecular dynamics simulations. Journal of computational chemistry 2009, 30, 2157-2164.

(39) Abraham, M. J.; Murtola, T.; Schulz, R.; Páll, S.; Smith, J. C.; Hess, B.; Lindahl, E. GROMACS: High performance molecular simulations through multi-level parallelism from laptops to supercomputers. SoftwareX 2015, 1, 19-25.

(40) Berendsen, H. J.; Postma, J. v.; van Gunsteren, W. F.; DiNola, A.; Haak, J. R. Molecular dynamics with coupling to an external bath. The Journal of chemical physics $\mathbf{1 9 8 4 ,}$ $81,3684-3690$. 
(41) Evans, D. J.; Holian, B. L. The nose-hoover thermostat. The Journal of chemical physics 1985, 83, 4069-4074.

(42) Essmann, U.; Perera, L.; Berkowitz, M. L.; Darden, T.; Lee, H.; Pedersen, L. G. A smooth particle mesh Ewald method. The Journal of chemical physics 103, 8577-8593.

(43) Hess, B.; Bekker, H.; Berendsen, H. J.; Fraaije, J. G. LINCS: a linear constraint solver for molecular simulations. Journal of computational chemistry 1997, 18, 1463-1472.

(44) Hagberg, A.; Swart, P.; S Chult, D. Exploring network structure, dynamics, and function using NetworkX; 2008.

(45) Han, K.; Venable, R. M.; Bryant, A.-M.; Legacy, C. J.; Shen, R.; Li, H.; Roux, B.; Gericke, A.; Pastor, R. W. Graph-theoretic analysis of monomethyl phosphate clustering in ionic solutions. The Journal of Physical Chemistry B 2018, 122, 1484-1494.

(46) Velinova, M.; Sengupta, D.; Tadjer, A. V.; Marrink, S.-J. Sphere-to-rod transitions of nonionic surfactant micelles in aqueous solution modeled by molecular dynamics simulations. Langmuir 2011, 27, 14071-14077.

(47) Ozkanlar, A.; Zhou, T.; Clark, A. E. Towards a unified description of the hydrogen bond network of liquid water: A dynamics based approach. The Journal of chemical physics 2014, 141, 214107.

(48) Takes, F. W.; Kosters, W. A. Computing the eccentricity distribution of large graphs. Algorithms 2013, 6, 100-118.

(49) Michaud-Agrawal, N.; Denning, E. J.; Woolf, T. B.; Beckstein, O. MDAnalysis: a toolkit for the analysis of molecular dynamics simulations. Journal of computational chemistry 2011, 32, 2319-2327.

(50) Erlinger, C.; Gazeau, D.; Zemb, T.; Madic, C.; Lefrancois, L.; Hebrant, M.; Tondre, C. Effect of nitric acid extraction on phase behavior, microstructure and interac- 
tions between primary aggregates in the system dimethyldibutyltetradecylmalonamide

(DMDBTDMA)/n-dodecane/water: A phase analysis and small angle X-ray scattering (SAXS) characterisation study. Solvent extraction and ion exchange 1998, 16, 707-738.

\section{Acknowledgement}

This work was supported by the U.S. Department of Energy (DOE), Basic Energy Sciences, Chemical Sciences, Geosciences, and Biosciences Division under contract DEAC0206CH11357. This research used resources from the Center for Institutional Research Computing at Washington State University. B.S thanks Bhabha Atomic Research Centre (BARC), Mumbai, India for granting extra-ordinary leave to work at Washington State University.

\section{Supporting Information Available}

Simulation snapshots, cluster analysis details under $\mathbf{C} 1$ and $\mathbf{C} 2$ conditions, cluster analysis results for studied systems, extended aggregation between water and acid molecules, shortrange non-bonded interaction energy vs. simulation time plots, oxygen-oxygen RDF profiles of water molecules, diffusion coefficient and SASA results. 